\title{
Robust 3D indoor positioning system based on radio map using Bayesian network
}

\begin{abstract}
Indoor positioning remains a serious issue due to the difficulty in attaining sufficient accuracy within an indoor environment using tracking technology of low complexity. Currently, most positioning systems do not embed the off-the-shelf (OTS) system which allows mobile devices to estimate location without using any additional hardware. In this paper, we propose a robust 3D indoor positioning system that is suitable for an indoor IoT application. This system based on Bayesian network that depends on Wi-Fi signals strength. It was experimentally tested in a building with pre-deployed access points (APs). The experimental results indicate that localization accuracy of the proposed system is high with the use of a small-sized radio map.
\end{abstract}

Keyword: Bayesian network; Positioning; Fingerprinting 